\title{
Therapy Object
}

National Cancer Institute

\section{Source}

National Cancer Institute. Therapy Object. NCI Thesaurus. Code C41174.

Pre-clinical trials experiment conducted on the animal model with the goal to reduce cancer burden. 